\title{
MAMÍFEROS PLEISTOCENOS DEL OESTE DE LA REGIÓN PAMPEANA, ARGENTINA
}

\author{
Alfredo E. ZURITA ${ }^{1}$, Germán M. GASPARINI ${ }^{1}$, \\ Esteban SOIBELZON ${ }^{1}$, María A. ALCARAZ ${ }^{2} y$ \\ Ángel R. MIÑO-BOILINI
}

\footnotetext{
${ }^{1}$ Departamento Científico Paleontología de Vertebrados. Facultad de Ciencias Naturales y Museo (UNLP). Paseo del Bosque s/n. (1900) La Plata, Buenos Aires. CONICET. Correo electróncio: aezurita74@yahoo.com.ar

${ }^{2}$ Departamento de Arqueología. Facultad de Ciencias Naturales y Museo (UNLP). Paseo del Bosque s/n. (1900) La Plata, Buenos Aires. ANPCYT. ${ }^{3}$ Secretaría General de Ciencia y Técnica. Cátedra de Paleontología. Facultad de Ciencias Exactas y Naturales y Agrimensura. Universidad Nacional del Nordeste y Centro de Ecología Aplicada del Litoral (CECOAL-CONICET).
}

Zurita, A. E., Gasparini, G. M., Soibelzon, E., Alcaraz, A. \& Miño-Boilini, A. R. 2007. Mamíferos pleistocenos del oeste de la región Pampeana, Argentina. [Pleistocene mammals of the western Pampean region, Argentina.] Revista Española de Paleontología, 22 (1), 77-87. ISSN 0213-6937.

\begin{abstract}
In southern South America, the basic knowledge of the Pleistocene palaeofauna comes from the eastern area of the Pampean region, specifically restricted to the actual territory of the Buenos Aires province. In this contribution we report an association of Pleistocene mammals comes from the western area of the Pampean region (San Francisco, Córdoba province). This association, composed basically by taxa adapted to the cold an arid environment present in the later Pleistocene, is compatible with a glacial moment. From a biostratigraphic point of view, it is important to remark the presence of the Equidae Equus (Amerhippus) neogeus and the Glyptodontidae Neosclerocalyptus paskoensis, both taxa limited to the Lujanian Stage (ca. 130-8 kyr BP). Finally, and from a taxonomic point of view, this association of Pleistocene mammals is similar to that developed in the eastern area of the pampean region, studied from the middle of the XIX century.
\end{abstract}

Key words: Argentina, Buenos Aires and Córdoba provinces, Pampean region, Lujanian, Pleistocene.

\section{RESUMEN}

En el extremo meridional de América del Sur, la mayor parte del conocimiento paleomastozoológico proviene del sector este de la región Pampeana, más precisamente del ámbito de la provincia de Buenos Aires. En esta contribución se da a conocer un conjunto de mamíferos pleistocenos provenientes del extremo oeste de la región Pampeana (San Francisco, provincia de Córdoba). En el conjunto de taxones estudiados (compuesto por elementos pampeano-patagónicos adaptados a los climas áridos y fríos del Pleistoceno más tardío) resulta significativa la presencia del Equidae Equus (Amerhippus) neogeus y del Glyptodontidae Neosclerocalyptus paskoensis, ya que permite acotar la antigüedad al Lujanense ( $c a .130-8$ ka AP). Esta asociación es congruente con el desarrollo de un período glacial. Taxonómicamente, este conjunto de mamíferos resulta compatible con aquel conocido para el sector oriental de la región Pampeana, intensamente estudiada desde mediados del siglo XIX.

Palabras clave: Argentina, Provincias de Buenos Aires y Córdoba, Región Pampeana, Lujanense, Pleistoceno.

\section{INTRODUCCIÓN}

Entre los países de América del Sur, la Argentina constituye uno de los de mayor tradición en la Paleontología de vertebrados (Reig, 1961). Así, la mayoría de los registros de vertebrados pleistocenos ( $c a$. 1800-8 ka AP) del extremo meridional de América del Sur proviene de yacimientos ubicados en el actual territorio de Argentina (Tonni \& 
Cione, 1999), y más precisamente de la región Pampeana, en el ámbito de la provincia de Buenos Aires (ver Tonni \& Scillato-Yané, 1997; Cione et al., 1999; Tonni et al., 1999a). En los últimos años, algunos autores han dado a conocer ciertas asociaciones paleofaunísticas pleistocenas extra-pampeanas, particularmente de las provincias de Corrientes (Álvarez, 1974; Scillato-Yané et al., 1998; Herbst \& Santa Cruz, 1999; Noriega et al., 2000; Zurita \& Lutz, 2002; Alcaraz \& Carlini; 2003; Tonni et al., 2005; Gasparini \& Zurita, 2005), Entre Ríos (Vucetich et al., 2001, 2005; Carlini et al., 2002, 2004; Noriega et al., 2004; Alcaraz et al. 2005), Chaco (Zurita 2000, 2002; Zurita \& Carlini, 2001; Zurita et al., 2004), Formosa (Tonni \& Scillato-Yané, 1997; Alcaraz \& Zurita, 2004; Prevosti et al., 2005) y Santa Fe (De la Fuente, 1997, 1999; Zurita et al., 2002).

La evidencia biogeográfica más significativa muestra una diferenciación paleofaunística y paleoambiental entre la región Mesopotámica (particularmente Entre Ríos y Corrientes) por un lado, y el territorio que abarcan las actuales provincias de Formosa, Chaco, norte de Santa Fe y la región Pampeana (ver Carlini et al., 2004).

En este contexto, el conocimiento que se tiene de la fauna pleistocena de la provincia de Córdoba es escaso, y la mayoría de los trabajos data de más de sesenta años de antigüiedad (Ameghino, 1889; Castellanos, 1925, 1944; Frenguelli, 1921). Una excepción a esto lo constituyen los aportes de Tauber (1997, 1999, 2000), Goya \& Tauber (2001), Tauber \& Di Ronco (2000, 2003), Cruz \& Bargo (2003) y Goya et al. (2003).

El objetivo de la presente contribución es dar a conocer un conjunto de mamíferos pleistocenos provenientes de los alrededores de la localidad de San Francisco (31 $26^{\circ} \mathrm{S}$ y $\left.62^{\circ} 05^{\prime} \mathrm{O}\right)$, provincia de Córdoba, Argentina.

Abreviaturas. AGM: Archivo Gráfico y Museo Histórico de San Francisco y la Región.

\section{SISTEMÁTICA PALEONTOLÓGICA}

Superorden XENARTHRA Cope, 1889

Orden CINGULATA Illiger, 1811

Superfamilia Glyptodontoidea Gray, 1869

Familia Glyptodontidae Gray, 1869

Subfamilia Glyptodontinae Gray, 1869

Género Glyptodon Owen, 1838

\section{Glyptodon cf. G. reticulatus Owen, 1845}

Material referido: gran porción de la región dorsal de la coraza asociada a parte de la cintura pélvica (AGM 009).

Comentarios: la asignación del material a esta especie se debe a que la parte de coraza preservada presenta un reticu- lado en el cual es difícil diferenciar la figura central de las periféricas, tal como lo describen Ameghino (1889: 785) y Duarte (1997: 353) en la caracterización de este taxón. La distribución estratigráfica se restringe al Bonaerense y Lujanense (ca. 500-8 ka AP) (Scillato-Yané et al., 1995; Carlini \& Scillato-Yané, 1999). El género Glyptodon es característico de ambientes abiertos con pastizales, de clima templado o templado frío (Scillato-Yané et al., 1995), al tiempo que habría sido un activo pastador (ver Fariña \& Parietti, 1983; Pérez et al., 2000). Desde una perspectiva biogeográfica Glyptodon es uno de los Glyptodontidae con mayor distribución latitudinal, que se extiende con seguridad desde el sur de Argentina hasta, probablemente, Venezuela (Marshall et al., 1984; Tonni \& Scillato-Yané, 1997). Los Glyptodontinae constituyen probablemente los únicos Glyptodontidae involucrados en el "Gran Intercambio Biótico Americano", dando origen en América del Norte al género Glyptotherium Osborn, 1902 (Gillette \& Ray, 1981).

Subfamilia Hoplophorinae Huxley, 1864

Tribu Hoplophorini Huxley, 1864

Género Neosclerocalyptus Paula Couto, 1957

\section{Neosclerocalyptus paskoensis (Zurita, 2002)} Fig. 2a, b y c

Material referido: han sido hallados tres ejemplares. Uno está representado por el cráneo completo, escudete cefálico, porciones laterales de la coraza dorsal, anillos y tubo caudal (AGM 002). El otro resto consiste en un cráneo parcialmente completo y mandíbula correspondiente a un individuo juvenil (AGM 006), mientras que el tercer ejemplar consta de una gran porción de coraza dorsal, tubo caudal y mandíbula (AGM 007).

Comentarios: Neosclerocalyptus es un género que cuenta con numerosas especies, la mayoría reconocidas por Gervais \& Ameghino (1880), Ameghino (1888, 1889, 1895) y Castellanos (1925), sobre la base de detalles en la ornamentación de los osteodermos y del tubo caudal (ver Hoffstetter, 1958; Paula Couto, 1979; Zurita et al., 2005); aunque no todas son consideradas actualmente como válidas (Carlini \& Scillato-Yané, 1999; Zurita et al., 2005). El registro de este género se extiende con seguridad desde el Ensenadense (Plioceno tardío-Pleistoceno medio) hasta el Lujanense (Pleistoceno tardío-Holoceno temprano) (Scillato-Yané et al., 1995; Carlini \& Scillato-Yané, 1999; Zurita et al., 2005). La única especie Lujanense reconocida (ver Zurita et al., 2005) está claramente adaptada a ambientes abiertos, áridos a semiáridos y fríos, y su presencia resulta escasa en aquellas áreas que desarrollaron procesos climático-ambientales más húmedos y cálidos, al menos durante parte del Pleistoceno (e.g. región Mesopotámica, sector occidental de Uruguay y sur de Brasil) (Noriega et al., 2004; Carlini et al., 2004). A latitudes más septentrionales, Neosclerocalyptus es reemplazado por otro Glyptodontidae Hoplophorini, Hoplophorus Lund, 1839, un 
taxón limitado hasta el momento al Estado de Minas Gerais, Brasil (Lagoa Santa; $19^{\circ} \mathrm{S}$ ) y al sector centro-sur de Bolivia (Tarija; 21 $33^{\prime} \mathrm{S}$ y $64^{\circ} 46^{\prime} \mathrm{O}$ ) (Paula Couto, 1957; Hoffstteter, 1964).

Superfamilia Mylodontoidea Gill, 1872

Familia Mylodontidae Gill, 1872

Subfamilia Scelidotheriinae Ameghino, 1904

Género Scelidotherium Owen, 1839

\section{Scelidotherium cf. S. leptocephalum Owen, 1839}

Material referido: mandíbula incompleta; con los molariformes m1-m3 derechos y m2-m4 izquierdos (AGM 014).

Comentarios: Estos molariformes, comparados con aqué- llos figurados por Ameghino (1889), Pascual et al. (1966) y McDonald (1987), como Scelidotherium leptocephalum, muestran la misma y típica conformación elíptico-prismática. El género Scelidotherium Owen, 1839 tiene su primer registro en el Vorohuense (Plioceno tardío), en tanto que $S$. leptocephalum es exclusivo del Lujanense (Scillato-Yané et al., 1995; Carlini \& Scillato-Yané, 1999), aunque McDonald (1987) extiende el primer registro de esta especie al Ensenadense al tiempo que aclara que poseen menor talla que las especies del Lujanense. Desde una perspectiva paleoambiental este taxón indica la presencia de ambientes abiertos con climas templados (Bombin, 1976). En cuanto a la distribución geográfica, los restos mejor conocidos provienen de la región Pampeana, Argentina (McDonald, 1987). Para el Uruguay, la asignación es dudosa (Ubilla \& Perea, 1999), en tanto que para Perú fue mencionado
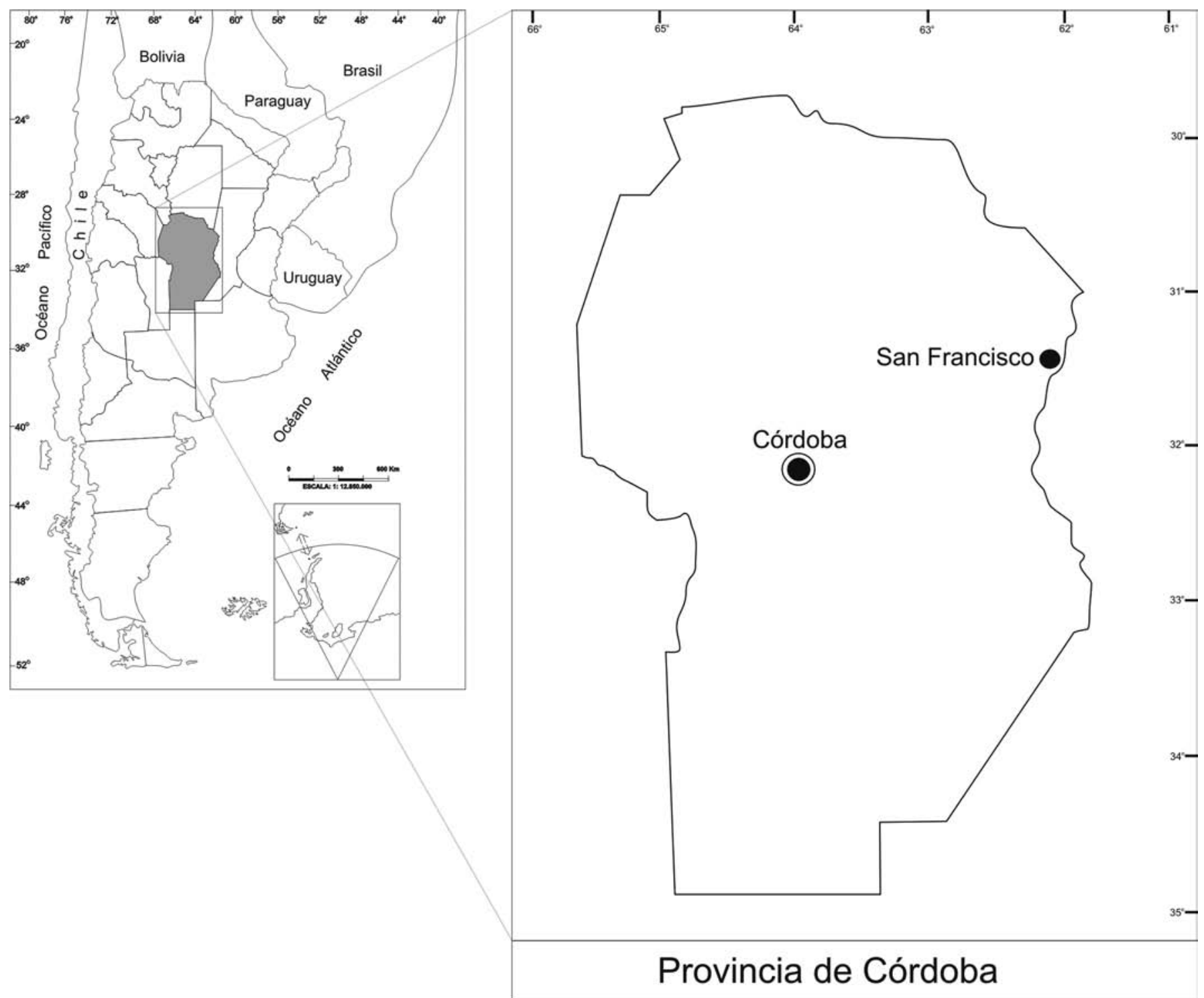

Figura 1. Mapa de ubicación. Location map. 
por Spillman (1949) y Pascual \& Odreman Rivas (1973), aunque estos restos son fragmentarios y aislados.

Orden CARNIVORA Bowdich, 1821

Superfamilia Feloidea Simpson, 1931

Familia Felidae Gray, 1821

Subfamilia Machairodontinae Gill, 1872

Género Smilodon Lund, 1842

\section{Smilodon populator Lund, 1842}

Fig. 2e

Material referido: esqueleto completo en buen estado de preservación (AGM 005).

Comentarios: Smilodon populator se caracteriza por poseer una amplia distribución cronológica y geográfica, pues se registra en el Ensenadense, Bonaerense y Lujanense ( $c a$. 1800-8 ka AP) de Argentina, Brasil, Bolivia (Paula Couto, 1979; Cione et al., 1999), Chile (Casamiquela, 1999), y Uruguay (Ubilla, 1996; Ubilla \& Perea, 1999). En Argentina es significativa su presencia en el área bonaerense, hallándose también en sedimentos cuaternarios de Córdoba, Entre Ríos y Santa Fe (Berman, 1994). Anatómicamente, uno de los rasgos más característicos de este Felidae es la hipertrofia de los caninos superiores, transversalmente comprimidos (Berman, 1994). Para ciertos autores (e.g. Berta, 1987) Smilodon parece haber sido un predador asociado a ambientes de vegetación arbórea, mientras que para otros (Kurtén \& Werdelin, 1990) este taxón podría haber estado adaptado a ambientes abiertos.

\section{Infraorden CYNOIDEA Flower, 1869 \\ Familia Canidae Fischer, 1817 \\ Género Dusicyon Hamilton-Smith, 1839}

\section{Dusicyon sp.}

Fig. 2d

Material referido: cráneo en buen estado de preservación pero sin la región occipital ni los arcos cigomáticos y premaxilar, con la mandíbula asociada (AGM 018).

Comentarios: La distribución estratigráfica del género abarca desde el Vorohuense (Plioceno tardío) hasta la actualidad. Los requerimientos ecológicos de este género se relacionan con un paisaje abierto, donde la comunidad de pastizales parece ser dominante (Berman, 1994). Al presente, este taxón incluye tres especies (D. griseus, $D$. culpaeus y $D$. gymnocercus) que poseen una alta tolerancia ecológica, puesto que habitan en una amplia variedad de ambientes (ver Redford \& Eisenberg, 1992).

Orden PERISSODACTYLA Owen, 1848

Superfamilia Equoidea Gray, 1821

Familia Equidae Gray, 1821

Subfamilia Equinae Steinmann y Doderlein, 1890 Género Equus Linné, 1758

$$
\begin{gathered}
\text { Equus (Amerhippus) cf. E. (A.) neogeus } \\
\text { Lund, } 1840 \\
\text { Fig. } 2 \mathrm{f}
\end{gathered}
$$

Material referido: $\mathrm{p} 4$ derecho (AGM 010).

Comentarios: La morfología de este material coincide con aquella referida por Pascual et al. (1966) a Equus (Amerhippus) neogeus. En el doble lazo, compuesto por el metacónido y el metastílido, el primero resulta redondeado y el segundo afecta una forma angular (ver Carlini \& Tonni, 2000). En líneas generales, los équidos resultan excelentes indicadores paleoambientales (Alberdi et al., 2003). E. (A.) neogeus es la especie de mayor gracilidad del género (Alberdi \& Prado, 1992, 1995; Alberdi et. al., 2003) y su distribución estratigráfica está restringida al Lujanense (Pleistoceno tardío-Holoceno temprano) de Argentina, Uruguay y Brasil. El ambiente inferido para el género abarca áreas abiertas de pastizales y estepas xerófilas, e incluso áreas abiertas de altura (Deschamps \& Tonni, 1992; Alberdi \& Prado, 1995; Alberdi et al., 2003).

Orden NOTOUNGULATA Roth, 1903

Familia Toxodontidae Gervais, 1847

Género Toxodon Owen, 1837

\section{Toxodon sp.}

Material referido: un incisivo superior (AGM 011).

Comentarios: Para el Pleistoceno medio-Holoceno temprano (Bonaerense-Lujanense) se reconocen, en el ámbito

Figura 2. a-c, Neosclerocalyptus paskoensis (Zurita, 2002); a, cráneo en vista lateral; b, escudete cefálico en vista dorsal; c, cráneo en vista lateral de un individuo juvenil. d, Dusycion sp., cráneo en vista lateral. e, Smilodon populator Lund, 1842, cráneo en vista lateral. f, Equus (Amerhippus) cf. E. (A.) neogeus Lund, 1840, p4 derecho en vista oclusal. Escala a-e: 10cm; escala f: $1 \mathrm{~cm}$.

$\boldsymbol{a}-\boldsymbol{c}$, Neosclerocalyptus paskoensis (Zurita, 2002); $\boldsymbol{a}$, skull in lateral view; $\boldsymbol{b}$, head-shield in dorsal view; , skull in lateral view belonging to a juvenile individual. , Dusycion sp., skull in lateral view. e, Smilodon populator Lund, 1842, skull in lateral view. $f$, Equus (Amerhippus) $c f$. E. (A.) neogeus Lund, 1840, p4 in oclusal view. Scale bar a-e: 10cm; scale bar f: $1 \mathrm{~cm}$. 


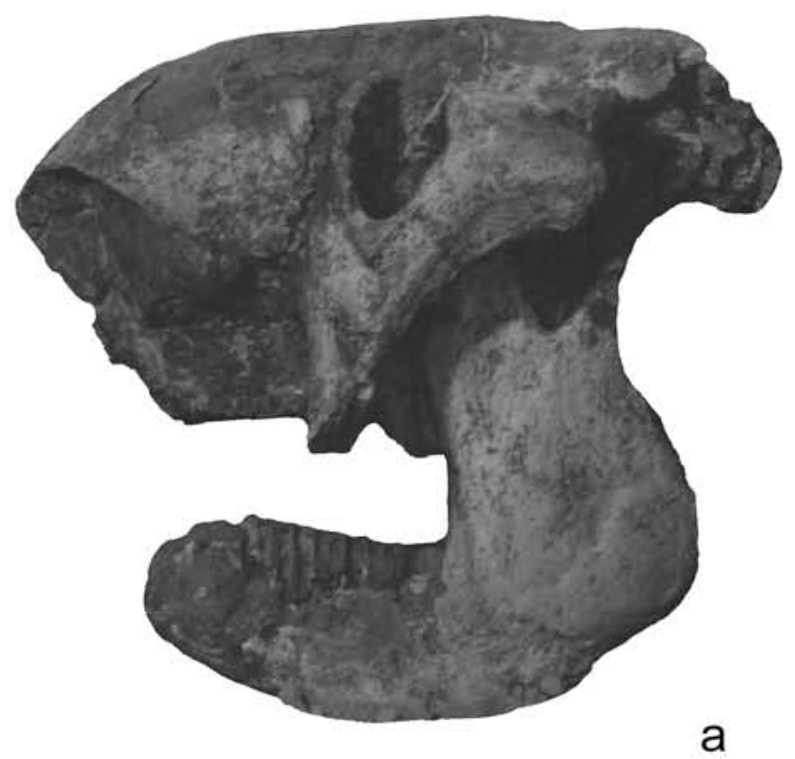

a

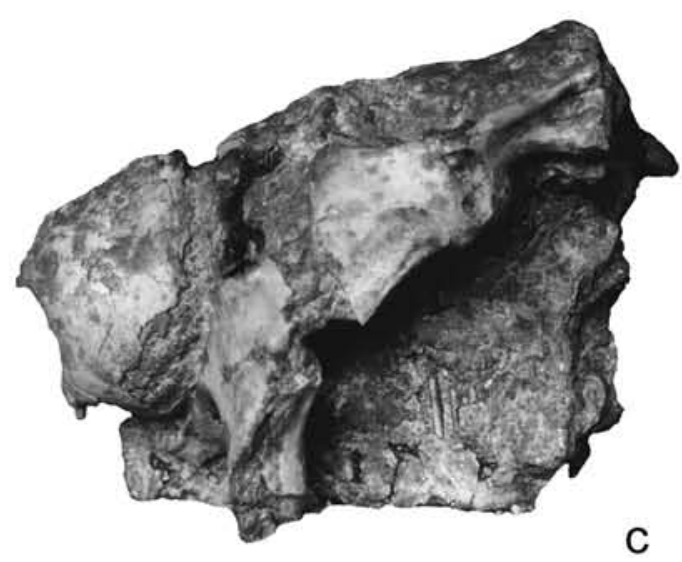

C

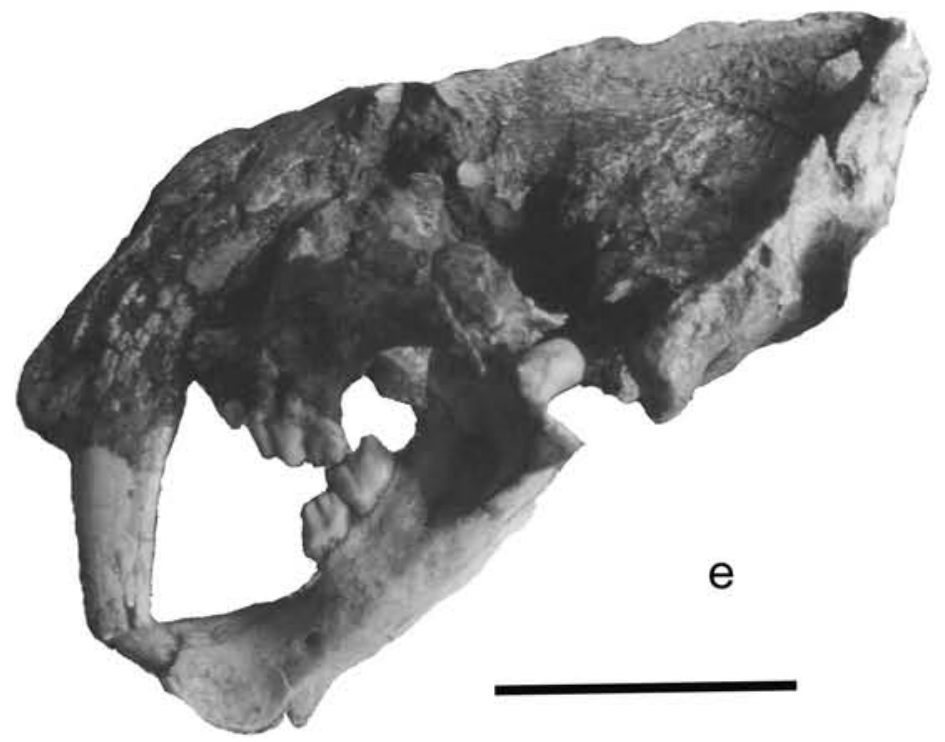

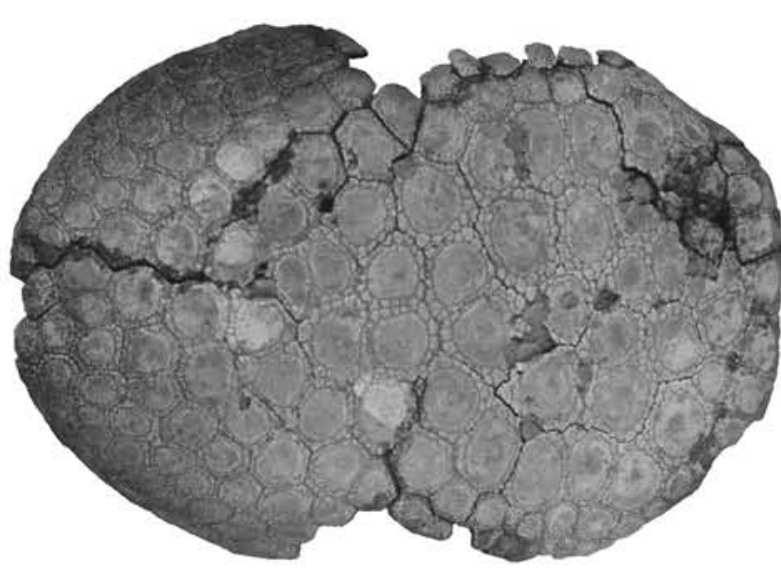

b
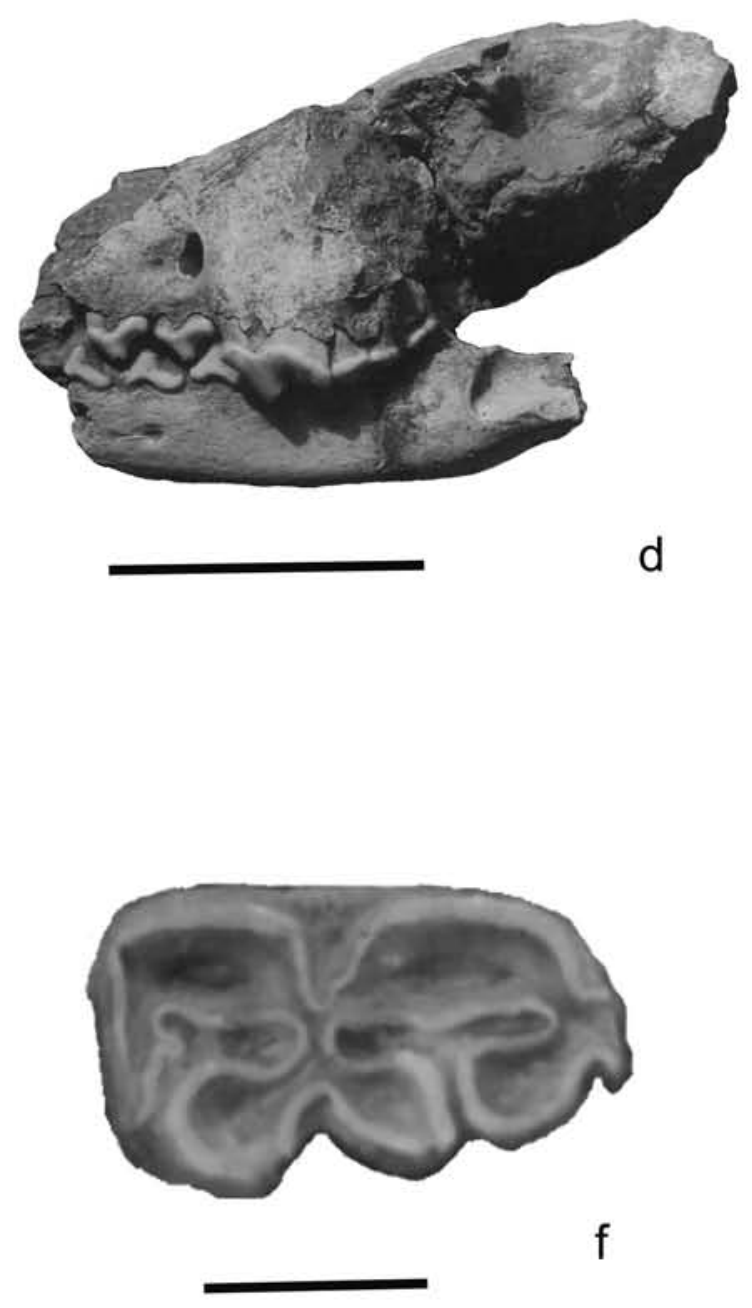
del territorio de Argentina, tres especies asignables al género Toxodon: T. platensis Owen, 1837, T. gezi C. Ameghino, 1917 y T. gracilis Gervais \& Ameghino, 1880. En este sentido, es de destacar que algunos autores creen que $T$. platensis y $T$. gezi podrían representar una única especie, ya que no es posible observar diferencias morfológicas significativas (ver Bond, 1999; Miño-Boilini et al., 2006). Apoyando esta hipótesis, una reciente revisión taxonómica efectuada por Pérez García (2004) sugiere la presencia, en el Pleistoceno de Argentina y de Uruguay, de una sola especie, T. platensis. Por otro lado, el estatus de T. gracilis permanece aún incierto, por cuanto se conocen pocos restos y estos provienen de un ámbito paleozoogeográfico particular (ver Miño-Boilini et al., 2006).

Estudios biomecánicos (Archuby, 1998) sugieren una vida cercana a cuerpos de agua. En general se considera a estas especies como formas pesadas y fundamentalmente pastadoras (Ubilla, 1985; Bond et al., 1995; Bond, 1999), por lo que se infiere, a partir de su presencia, la existencia de ambientes abiertos. La escasez del material aquí estudiado impide una asignación específica.

\section{Orden ARTIODACTYLA Owen, 1848 \\ Suborden TYLOPODA, 1811 \\ Subfamilia Camelinae Zittel, 1893 \\ Tribu Lamini Webb, 1974}

Género Hemiauchenia H. Gervais \& Ameghino, 1880

\section{Hemiauchenia paradoxa \\ H. Gervais \& Ameghino, 1880}

Material referido: fémur derecho (AGM 019).

Comentarios: Dentro de los camélidos extintos, el género Hemiauchenia corresponde a una forma de gran talla, perteneciente al extremo superior del rango de tamaño conocido para los integrantes sudamericanos del grupo (Menégaz \& Ortiz Jaureguizar, 1995). Su distribución cronológica en Argentina abarca desde el Ensenadense (Plioceno tardíoPleistoceno medio), con restos provenientes de las clásicas "Toscas del Río de La Plata" (en localidad de Anchorena, provincia de Buenos Aires) hasta el Lujanense (Pleistoceno tardío-Holoceno temprano) de la región pampeana (Menégaz \& Ortiz Jaureguizar, op. cit.). Este género, que tiene además registros en Uruguay (Formación Sopas; Ubilla \& Perea, 1999) y en el Sur de Brasil (Oliveira, 1999), cuenta hasta el momento con una única especie reconocida, $H$. paradoxa (ver Menégaz, 2000).

La asignación del material a esta especie se debe principalmente a su tamaño, ya que sus dimensiones se ajustan al rango conocido para Hemiauchenia, considerada una de las formas gigantes de la familia (Menégaz, 2000). Desde una perspectiva ecológica, se establece a $H$. paradoxa como una forma pastadora, adaptada a ambientes abiertos de pastizales (Menégaz \& Ortiz Jaureguizar, 1995), segu- ramente bajo condiciones áridas o semiáridas, teniendo en cuenta los requerimientos de los representantes actuales de la familia, algunos de ellos indicadores de ambientes xéricos (ver Redford \& Eisenberg, 1992).

\section{DISCUSIÓN DE LOS RESULTADOS}

En este conjunto paleofaunístico resulta bioestratigráficamente significativa la presencia del Equidae Equus (Amerhippus) cf. E. (A.) neogeus y del Glyptodontidae Neosclerocalyptus paskoensis, ya que ambos permiten acotar la antigüedad de esta asociación al Lujanense (Pleistoceno tardío-Holoceno temprano, 130-8 ka AP; Alberdi et al., 2003; Zurita et al., 2005; Cione \& Tonni, 2005).

En la región Pampeana, el Lujanense está bioestratigráficamente sustentado en la biozona de $E$. (A.) neogeus (Cione \& Tonni, 1995, 1999). En el ámbito de la provincia de Buenos Aires, el Pleistoceno más tardío está representado por la base del Miembro La Chumbiada y el Miembro Guerrero de la Formación Luján, incluyendo a la ingresión marina producida por el último interglacial (OIS 5e) (ver Pardiñas et al., 1996; Cione \& Tonni, 1999; Tonni et al., 1999b). La sedimentación de esta secuencia abarca un lapso aproximado de $28.900 \pm 400$ y 10.000 AP (ver Prado et al., 2001; Tonni et al, 2003). El Miembro Guerrero (ca. 21-10 ka AP) y su fauna representan varios eventos desde el último máximo glacial, pasando por el Bolling y llegando al último avance glacial (Convergencia Fría Antártica más Younger Dryas). Todo esto estaría reflejado en la fauna (e.g. presencia del roedor Lundomys en Paso Otero) (Tonni et al., 2003).

En este escenario climático-ambiental, todos los taxones aquí analizados son compatibles con la fauna Lujanense del sector este de la región pampeana, compuesta básicamente por elementos pampeano-patagónicos adaptados a los ambientes fríos y áridos imperantes durante la mayor parte del Pleistoceno tardío como Neosclerocalyptus paskoensis, Equus (A.) neogeus, Hemiauchenia paradoxa (ver Tonni et al., 1999b; Prado et al., 2001). Así, esta asociación es congruente con un período glacial, involucrando posiblemente, el último estadio glacial, que incluye asimismo al último máximo glacial (ca. 18 ka AP).

En este sentido, diversos autores (e.g. Tauber, 1999; Goya \& Tauber, 2001; Goya et al., 2003; Tauber \& Di Ronco, 2003; Cruz \& Bargo, 2003) han reportado dentro del ámbito de la provincia de Córdoba otras asociaciones de mamíferos de antigüedad Lujanense, algunas con dataciones absolutas [e.g. Lama (Vicugna) aff. L. (V.) gracilis: 21 ka AP; Glyptodon reticulatus: 40.830 años AP (ver Tauber, 1997; Tauber \& Di Ronco, 2000)]. Como en la asociación aquí estudiada, el conjunto de taxones indica el predominio de ambientes áridos o semiáridos (e.g. Ctenomys, Lagostomus, Lama (Vicugna) aff. L. (V.) gracilis; ver Redford \& Eisenberg, 1992; Tonni et al., 1999b; 
Tauber, 1999; Menégaz, 2000) con pastizales o vegetación de estepa. Este escenario es congruente con la hipótesis propuesta por Iriondo \& García (1993), que sostiene una prolongación de las condiciones climático-ambientales presentes actualmente en la Patagonia, $750 \mathrm{~km}$ al noreste de Argentina, durante el Pleistoceno más tardío.

Mac Fadden \& Shockey (1997) han propuesto que algunos "megamamíferos" (e.g. Toxodon platensis, Macrauchenia patachonica, Cuvieronius) tradicionalmente considerados pastadores, podrían haber actuado alternativamente como ramoneadores. Esto podría sugerir la presencia de ambientes subarborizados, ya que estos taxones han sido reportados en sedimentos del Pleistoceno tardío de la provincia de Córdoba (ver Goya \& Tauber, 2003; Cruz \& Bargo, 2003). Apoyando esta hipótesis, Carignano (1996) ha registrado para el Pleistoceno tardío-Holoceno temprano de Córdoba ( $c a$. 5-9 ka AP), la predominancia de climas secos y áridos o semiáridos, en donde podrían haberse intercalado ciertos períodos húmedos y subhúmedos (entre 50-30 ka AP), representados por una interrupción en la sedimentación loéssica (ver también Kröhling \& Iriondo, 1999).

De modo tentativo, los mamíferos exhumados en los alrededores de la localidad de San Francisco, Córdoba, podrían provenir de la Formación Tezanos Pinto, cuya sedimentación abarca entre 36-8 ka AP y está compuesta básicamente por loess, con un marcado predominio de la fracción limo (ver Kröhling, 1998; Kröhling \& Orfeo, 2002).

Este marco faunístico (y climático-ambiental) se habría extendido hacia el norte, abarcando el centro-norte de Argentina, Paraguay y el sudeste de Bolivia (Hoffstetter, 1978; Tonni \& Scillato-Yané, 1997; Carlini \& Tonni, 2000; Carlini et al., 2004; Zurita et al., 2004). Por el contrario, hacia la Mesopotamia, la presencia de taxones de origen brasílico (e.g. Pteronura, Tapirus, Holmesina paulacoutoi; ver, entre otros, Tonni, 1992; Carlini et al., 2002; Scillato-Yané et al., 2005), conjuntamente con la escasa presencia de taxones adaptados a ambientes fríos y áridos (e.g. Neosclerocalyptus paskoensis; ver Zurita et al., 2005) y ciertas peculiaridades paleobiogeográficas (e.g. diferencias de tamaño corporal en Lama, Toxodon gracilis y Lestodon; ver Noriega et al., 2004) sugieren condiciones ambientales algo más húmedas y cálidas que aquellas inferidas para la región Pampeana (ver, entre otros, Clapperton, 1993; Tonni et al., 1999b; Cione \& Tonni, 2001; Prado et al., 2001; Tonni et al., 2003; Ubilla et al., 2004).

\section{CONCLUSIONES}

a) Desde una perspectiva bioestratigráfica, la presencia del Equidae Equus (A.) neogeus y del Glyptodontidae Neosclerocalyptus paskoensis permiten acotar la antigüedad de la asociación de mamíferos aquí estudiada al Luja- nense ( $c$ a. 130-8 ka AP). De modo tentativo, estos taxones podrían provenir de la Formación Tezanos Pinto (ca. 368 ka AP).

b) Esta asociación, compuesta por elementos pampeano-patagónicos, es congruente con un período glacial.

c) Desde una perspectiva climático-ambiental, la evidencia sugiere el desarrollo predominante de ambientes abiertos áridos o semiáridos, probablemente subarborizados. Este escenario es compatible con aquel inferido para el Lujanense del sector oriental de la región Pampeana.

\section{AGRADECIMIENTOS}

Los autores expresan su agradecimiento al Dr. E. P. Tonni por la lectura crítica del manuscrito y sus importantes comentarios. Al Dr. F. J. Prevosti por la determinación taxonómica de los materiales de cánidos y félidos. A las autoridades del Archivo Gráfico y Museo Histórico de San Francisco y la Región por haber cedido el material para su estudio, a los revisores Dr. J. L. Prado y al Dr. G. J. Scillato- Yané y a la Lic. Verónica Espíndola por la realización de las láminas.

\section{BIBLIOGRAFÍA}

Alberdi, M. T. \& Prado, J. L. 1992. El registro de Hippidion Owen, 1896 y Equus (Amerhippus) Hoffstetter, 1950 (Mammalia, Perissodactyla) en América del Sur. Ameghiniana, 29, 265-284.

Alberdi, M. T. \& Prado, J. L. 1995. Los Équidos de América del Sur. In: Evolución climática y biológica de la región Pampeana durante los últimos cinco millones de años. Un ensayo de correlación con el Mediterráneo occidental (eds. M. T. Alberdi, G. Leone \& E. P. Tonni). Museo Nacional de Ciencias Naturales, Consejo Superior de Investigaciones Científicas, Monografías, Madrid, 14, 295-308.

Alberdi, M. T., Cartelle, C. \& Prado, J. L. 2003. El registro de Equus (Amerhipus) e Hippidion (Mammalia, Perissodactyla) de Brasil. Consideraciones paleoecológicas y biogeográficas. Ameghiniana, 40, 173-196.

Alcaraz, M. A. \& Carlini, A. A. 2003. Los cérvidos (Mammalia, Artiodactyla) de las Formaciones Toropí y Yupoí (Pleistoceno medio-tardío) de la provincia de Corrientes, Argentina. Ameghiniana, 40, 50R.

Alcaraz, M.A., Ferrero, B.S. \& Noriega, J.I. 2005. Primer registro de Antifer ultra Ameghino, 1889 (Artiodactyla, Cervidae) en el Pleistoceno de Entre Ríos. Revista del Instituto Superior de Correlación Geología (Misceláneas), 14, 65-70.

Alcaraz, M. A. \& Zurita, A. E. 2004. Nuevos registros de cérvidos poco conocidos: Epieuryceros cf. proximus Castellanos y Antifer sp. (Mammalia, Artiodactyla, Cervidae). Revista del Museo Argentino de Ciencias Naturales, 6 , 41-48.

Álvarez, B. B. 1974. Los mamíferos fósiles del Cuaternario 
de Arroyo Toropí, Corrientes, Argentina. Ameghiniana, 11, 295-311.

Ameghino, C. 1917. Dos nuevas especies de Toxodontes. Physis, 3, 288.

Ameghino, F. 1888. Rápidas diagnosis de mamíferos fósiles nuevos de la República de Argentina. Obras completas, 5, 471-480.

Ameghino, F. 1889. Contribución al conocimiento de los mamíferos fósiles de la República Argentina. Actas de la Academia Nacional de Ciencias de Córdoba, Argentina, 6, 1-1027.

Ameghino, F. 1895. Sur les Edentés fosiles de l'Argentine (examen critique, revision et correction de l'ouvrage de M.R. Lydekker The extinct Edentates of Argentina). Revista del Jardin Zoológico de Buenos Aires, 3, 97-192.

Archuby, F. 1998. Alometría de los huesos largos de Toxodon platensis (Notoungulata) y Macrauchenia patachonica (Litopterna). Implicancias en su locomoción. $7^{\circ}$ Congreso Argentino de Paleontología y Bioestratigrafía, Bahía Blanca, Argentina, resúmenes, 101.

Berman, W. D. 1994. Los Carnívoros continentales (Mammalia, Carnívora) del Cenozoico en la provincia de Buenos Aires. Tesis Doctoral, Facultad de Ciencias Naturales y Museo, Universidad Nacional de La Plata, Buenos Aires, 1-452 (inédita).

Berta, A. 1987. The sabercat Smilodon gracilis Florida and discusión of its relationship (Mammalia, Felidae, Smilodontini). Bulletin Florida State Museum, Biological Sciences, 31, 1-63.

Bombin, M., 1976. Modelo Paleoecológico-evolutivo para o Neoquaternário da regiao da Campanha- Oeste do Rio Grande do Sul (Brasil). A Formaçao Touro Passo, seu conteúdo fossilífero e a pedogénese pós-deposicional. Comunicaçoes do Museu de Ciencias da PUCRGS, 15, 1-90.

Bond, M. 1999. Evolution of Quaternary Xenarthrans (Mammalia) of Argentina. In: Quaternary of South America and Antarctic Peninsula (Eds. J. Rabassa \& M. Salemme). Centro Austral de Investigaciones Científicas and Universidad Nacional de la Patagonia, Ushuaia, Tierra del Fuego, 12, 177-205.

Bond, M., Cerdeño, E. P. \& López, G., 1995. Los Ungulados nativos de América del Sur. In: Evolución climática y biológica de la región Pampeana durante los últimos cinco millones de años. Un ensayo de correlación con el Mediterráneo occidental (Eds. M. T. Alberdi, G. Leone \& E. P. Tonni). Museo Nacional de Ciencias Naturales, Consejo Superior de Investigaciones Científicas, Monografías, Madrid, 12, 257-275.

Carignano, C. A. 1996. Evolución geomorfológica de las planicies en la provincia de Córdoba durante el Pleistoceno superior. Revista del Instituto de Geología y Minería, 11, 7-26.

Carlini, A.A. \& Scillato-Yané, G. J. 1999. Evolution of Quaternary Xenarthrans (Mammalia) of Argentina In: Quaternary of South America and Antarctic Peninsula (Eds. J. Rabassa \& M. Salemme). Centro Austral de Investigaciones Científicas and Universidad Nacional de la Patagonia, Ushuaia, Tierra del Fuego, 12, 149-175.
Carlini, A. A. \& Tonni, E. P. 2000. Mamíferos Fósiles del Paraguay. Cooperación Técnica Paraguayo-Alemana. Proyecto Sistema Ambiental del Chaco-Proyecto Sistema Ambiental Región Oriental, Buenos Aires, 108 pp.

Carlini, A. A., Tonni, E. P. \& Noriega, J. I. 2002. El primer registro paleontológico del lutrino gigante Pteronura (Carnívora, Mustelidae) en la Argentina. Su importancia paleobiogeográfica. $1^{\circ}$ Congreso Latinoamericano de Paleontología de Vertebrados, Santiago de Chile, Chile, resúmenes, 25.

Carlini, A. A., Zurita, A. E., Gasparini, G. M. \& Noriega, J. I. 2004. Los mamíferos del Pleistoceno de la Mesopotamia argentina y su relación tanto con aquellos del Centro-Norte de la Argentina, Paraguay, sur de Bolivia, como con los del sur de Brasil y oeste de Uruguay: Paleobiogeografía y Paleoambientes. Revista del Instituto Superior de Correlación Geología (Misceláneas), 12, 5-12.

Casamiquela, R. 1999. The vertebrate record of Chile. In: Quaternary of South America and Antarctic Peninsula (eds. J. Rabassa \& M. Salemme), 7, 91-107.

Castellanos, A. 1925. Descripción de un tubo caudal de Sclerocalyptus matthewi n. sp. (Descubierto en el Pampeano inferior del Valle de los Reartes, Sierras de Córdoba). Revista de la Universidad Nacional de Córdoba, 10-12, 1- 54.

Castellanos, A. 1944. Paleontología estratigráfica de los sedimentos neógenos de la provincia de Córdoba. Publicaciones del Instituto de Fisiografía y Geología, 23, 1-47.

Cione, L. A. \& Tonni E. P. 1995. Bioestratigrafía y cronología del cenozoico de la región Pampeana. In: Evolución biológica y climática de la región Pampeana durante los últimos cinco millones de años. Un ensayo de correlación con el Mediterráneo occidental (eds. M. T. Alberti, G. Leone \& E.P. Tonni). Museo Nacional de Ciencias Naturales, Consejo Superior de Investigaciones Científicas, Monografías, 2, 47-74.

Cione A. L. \& Tonni E. P. 1999. Biostratigraphy and chronological scale of uppermost Cenozoic in the Pampean area, Argentina. In: Quaternary of South America and Antarctic Peninsula (Eds. J. Rabassa \& M. Salemme). Centro Austral de Investigaciones Científicas and Universidad Nacional de la Patagonia, Ushuaia, Tierra del Fuego, 3, 23-52.

Cione, A. L. \& Tonni, E. P. 2001. Correlation of Pliocene to Holocene southern South American and european vertebrate-bearing units. Bolletino della Societá Paleontologica Italiana, 40, 167-173.

Cione, A. L. \& Tonni, E. P. 2005. Bioestratigrafía basada en mamíferos del Cenozoico Superior de la provincia de Buenos Aires, Argentina. $16^{\circ}$ Congreso Geológico Argentino, Relatorio, 183-200.

Cione, L. A., Tonni E. P., Bond M., Carlini A. A., Pardiñas, U. F., Scillato-Yané, G. J., Verzi, D. \& Vucetich, M. G. 1999. Ocurrence charts of Pleistocene mammals in the Pampean area, eastern Argentina. In: Quaternary of South America and Antarctic Peninsula (Eds. J. Rabassa \& M. Salemme). Centro Austral de Investigaciones Científicas and Universidad Nacional de la Patagonia, Ushuaia, Tierra del Fuego, 12, 53-59. 
Clapperton, C. 1993. Nature of enviromental changes in South America at the Last Glacial Maximum. Palaeogeography, Palaeoclimatology, Palaecology, 101, 189-208.

Cruz, L. E. \& Bargo, S. 2003. Mamíferos del Pleistoceno tardío-Holoceno temprano del departamento Río Cuarto, Córdoba. Importancia bioestratigráfica. Ameghiniana, 40, 83 R.

De la Fuente, M. 1997. Las tortugas pleistocenas del extremo meridional de la provincia de Santa Fe, Argentina. Stvdia Geológica Salmaticensia, 33, 67-90.

De la Fuente, M. 1999. A review of the Pleistocene reptiles of Argentina: Taxonomic and palaeoenvironmental considerations. In: Quaternary of South America and Antarctic Peninsula (Eds. J. Rabassa \& M. Salemme). Centro Austral de Investigaciones Científicas and Universidad Nacional de la Patagonia, Ushuaia, Tierra del Fuego, 8, 109-136.

Deschamps, C. M. \& Tonni, E. P. 1992. Los vertebrados del Pleistoceno tardío del arroyo Napostá Grande, provincia de Buenos Aires. Aspectos paleoambientales. Ameghiniana, 29, 201-210.

Duarte, R. G. 1997. Gliptodontes del Pleistoceno tardío de Aguas de las Palomas, Campo de Pucará, Catamarca, Argentina. Variaciones morfológicas del caparazón de Glyptodon reticulatus Owen, 1845. Ameghiniana, 34, 345-355.

Fariña, R. A. \& Parietti, M. 1983. Uso del método RFTRA en la comparación de la morfología craneana en Edentata. $3^{\circ}$ Jornadas de Ciencias Naturales, resúmenes, 106-108.

Frenguelli, J. 1921. Sobre un Proterotérido del Pampeano superior de Córdoba. Neolicaphrium recens, nob. Actas de la Academia Nacional de Ciencias, 7, 7-23.

Gasparini, G. M. \& Zurita, A. E. 2005. Primer registro fósil de Tayassu pecari (Link) (Mammalia, Artiodactyla) en la Argentina. Ameghiniana, 42, 473-480.

Gillette, D. D. \& Ray, C. E. 1981. Glyptodonts of North America. Smithsonian Contributions to Palaeobiology, 40, 1-251.

Gervais, H. \& Ameghino, F. 1880. Los Mamíferos de la America del Sud. Igon Hermanos (eds.), Buenos Aires, 132-170.

Goya, F. \& Tauber, A. 2001. Mamíferos cuaternarios de una pampa de altura (Pampa de San Luis), departamento Cruz del Eje, provincia de Córdoba. Ameghiniana, 38, 10R.

Goya, F., Tauber, A. \& Cabrera, M. C. 2003. Yacimiento de mamíferos pleistocenos de la pampa Vaca Corral, departamento Cruz del Eje, Córdoba. Ameghiniana, 40, 86R

Herbst, R. \& Santa Cruz, J. N. 1999. Mapa litoestratigráfico de la provincia de Corrientes. D'Orbignyana, 2, 1-69.

Hoffstetter, R., 1958. Xenarthra. In : Traité de Paléontologie (ed. J. Piveteau), 6, 535-636.

Hoffstetter, R. 1964. Les Glyptodontes du Pléistocene de Tarija (Bolivie). I. Genres Hoplophorus et Panochthus. Bulletin de la Societé Geologique de France, sér. $7^{\mathrm{eme}}$, 7, 126- 133.

Hoffstetter, R. 1978. Une faune de Mammifères pléistocènes au Paraguay. Comptes Rendus Sommaires des Séances de la Societé Géologique de France, 1978, 32-33.
Iriondo, M \& García, N. O. 1993. Climatic variations in the Argentine plains during the last 18.000 years. $\mathrm{Pa}$ laeogeography, Palaeoclimatology, Palaeoecology, 101, 209-220.

Kröhling, D. M. 1998. Excursion Guide $n^{\circ} 2$ : North Pampa (Carcarañá river basin, Santa Fe province). Int. Joint Field Meeting "Loess in Argentina: Temperate and Tropical” (INQUA, PASH, CLIP, UNER), 33 pp.

Kröhling, D. M. \& Iriondo, M. 1999. Upper Quaternary of the Mar Chiquita area, North Pampa, Argentina. Quaternary International, 57/58, 149-163.

Kröhling, D. M \& Orfeo, O. 2002. Sedimentología de unidades loéssicas (Pleistoceno tardío-Holoceno) del centrosur de Santa Fe. Revista de la Asociación Argentina de Sedimentología, 9, 135-154.

Kurtén, B. \& Werdelein, L. 1990. Relationship between North and South American Smilodon. Journal of Vertebrate Palaeontology, 10, 158-169.

Lund, P.W. 1839. Coup da oeil sur les espéces éteintes de mammifères du Bresil, extrait de quelques mémoires presentés a la Acadamie royal des Sciences de Copenhaghe. Annales des Sciences Naturelles (Zoologie), 2, 214-234.

Lund, P.W. 1840. Blik paa Brasiliens Dyreverden für Sidste Jordomvaeltning tredie Afhandling: Forsaettlse af Pattedyrene. Det Kongelige Danske Videnskabernes Seskbas Naturvidenskabelige og Mathematiske Afhandlinger, 8 , 217-272.

Lund, P.W. 1842. Blik paa Brasiliens dyreverden für sidste Jordomvaeltning. Fjerde Afhandling, Fortsaettelse af Pattedryene, 9, 137-208.

MacFadden, B. J \& Shockey, B. J. 1997. Ancient feeding ecology and niche differentiation of Pleistocene mammalian herbivores from Tarija, Bolivia: morphological and isotopic evidence. Palaeobiology, 32, 77-100.

McDonald, H. G. 1987. A systematic review of the PlioPleistocene Scelidotherinae Ground Sloths (Mammalia, Xenarthra; Mylodontidae). Ph. D. Thesis, University of Toronto, 478 pp. (inédito).

Marshall, L., Berta, A., Hoffstetter, R., Pascual, R., Reig, R., Bombin, M. \& Mones, A. 1984. Mammals and stratigraphy: geochronology of the continental mammal-bearing Quaternary of South America. Palaeovertebrata, Mémoire Extraordinaire, 1-76.

Menégaz, A. N. 2000. Los Camélidos y Cérvidos del Cuaternario del Sector Bonaerense de la Región Pampeana. Tesis Doctoral, Universidad Nacional de La Plata, 240 pp. (inédita).

Menégaz, A. N. \& Ortiz Jaureguizar, E., 1995. Los artiodáctilos. In: Evolución climática y biológica de la región Pampeana durante los últimos cinco millones de años. Un ensayo de correlación con el Mediterráneo occidental (eds. M. T. Alberdi, G. Leone \& E. P. Tonni). Museo Nacional de Ciencias Naturales, Consejo Superior de Investigaciones Científicas, Monografías, Madrid, 15, 311-335.

Miño-Boilini, A.R. Cerdeño, E. \& Bond, M. 2006. Revisión del género Toxodon Owen, 1837 (Notoungulata: Toxo- 
dontidae) en el Pleistoceno de las provincias de Chaco, Corrientes y Santa Fe, Argentina. Revista Española de Paleontología, 21, 93-103.

Noriega, J. I., Manzano, A.S., de la Fuente, M.S. \& Tonni, E.P. 2000. Un Testudininae gigante (Chelonii: Criptodira) del Pleistoceno de la provincia de Corrientes, Argentina. Ameghiniana, 37, 321-326.

Noriega, J.I., Carlini, A.A. \& Tonni, E. P. 2004. Vertebrados del Pleistoceno tardío de la cuenca del arroyo Ensenada (Departamento Diamante, provincia de Entre Ríos, Argentina). Revista del Instituto Superior de Correlación Geología (Misceláneas), 12, 71-76.

Oliveira, E. 1999. Quaternary vertebrates and climates of southern Brasil. In: Quaternary of South America and Antarctic Peninsula (eds. J. Rabassa \& M. Salemme), 5, 61-73.

Owen, R. 1837. A description of the cranium of the Toxodon platensis, a gigantic extinct mammiferous species, referible by its dentition to the Rodentia, but with affinities to the Pachydermata and the herbivorous Cetacea. Geological Society of London, Proceedings, 2, 541-542.

Owen, R. 1839. Fossil Mammalia (3) In The Zoology of the voyage of H.H.M.S. Beagle. Smith, Elder and Co., London, 1 (8), 65-80.

Owen, R. 1845. Descriptive and illustrated catalogue of the fossil organic remains of Mammalia and Aves contained in the Museum of the Royal College of Surgeons of England. R. \& J. E. Taylor, London, 1-391.

Pardiñas, U.F.J., Gelfo, J.N., San Cristóbal, J. Cione, A.L. \& Tonni, E.P. 1996. Una asociación de organismos marinos y continentales en el pleistoceno superior en el sur de la provincia de Buenos Aires, Argentina $13^{\circ}$ Congreso Geológico Argentino y $3^{\circ}$ congreso de Explotación de Hidrocarburos, Buenos Aires, Actas, 5, 95-111.

Pascual, R. \& Odreman Rivas, O.E. 1973. Estudio del Material osteológico extraído de la Caverna de Huargo, departamento de Huanuco, Perú. In: Excavaciones en la Caverna de Huargo, Perú (ed. A. Cardich). Revista del Museo Nacional, 39, 31-39.

Pascual, R., Ortega Hinojosa, E. J., Gondar, D. G, \& Tonni E. P. 1966. Vertebrata. In: Paleontografía Bonaerense. Vertebrata I (ed. A. V. Borrello). Comisión de Investigaciones Científicas de la provincia de Buenos Aires, 1-202.

Paula Couto, C. de, 1957. Sôbre um gliptodonte do Brasil. Boletim Divisão de Geologia e Mineralogia. Rio de Janeiro, 165:1-37.

Paula Couto, J. C. 1979. Tratado de Paleomastozoología. Academia Brasileira de Ciencias, Río de Janeiro, 590 pp.

Pérez, L. M., Scillato-Yané, G. J. \& Vizcaíno, S. F. 2000. Estudio morfofuncional del aparato hiodeo de Glyptodon cf. clavipes (Cingulata: Glyptodontidae). Ameghiniana, 37, 293-299.

Pérez García, M. I. 2004. Ontogenia en toxodontinos avanzados, con base en el género Toxodon Owen, 1837. Tesis para optar a una Maestría en Biología, opción Zoología. Montevideo, Uruguay, 1-319 (inédita).

Prado, J. L., Alberdi, M. T., Azanza, B., \& Sanchez, B. 2001. Climate and changes in mammal diversity during the late
Pleistocene-Holocene in the Pampean Region (Argentina). Acta Paleontologica Polonica, 46, 261 -276.

Prevosti, F. J., Zurita, A. E. \& Carlini, A. A. 2005. Biostratigraphy, systematics and palaecology of Protocyon Giebel, 1855 (Carnivora, Canidae) in South America. Journal of South American Earth Sciences, 20, 5-12.

Redford, K.H. \& Eisenberg, J. F. 1992. Mammals of the Neotropics. The Southern Cone (Chile, Argentina, Paraguay and Uruguay). The University of Chicago Press, Chicago and London, 2, 1-432

Reig, O. 1961. La Paleontología de Vertebrados en la Argentina. Retrospección y perspectiva. Holmbergia, 6 (17), 1-127

Scillato-Yané, G. J., Carlini, A. A., Vizcaíno, S. F. \& Ortiz Jaureguizar, E. 1995. Los Xenarthros. In: Evolución climática y biológica de la región Pampeana durante los últimos cinco millones de años. Un ensayo de correlación con el Mediterráneo occidental (eds. M. T. Alberdi, G. Leone \& E. P. Tonni). Museo Nacional de Ciencias Naturales, Consejo Superior de Investigaciones Científicas, Monografías, Madrid, 9, 181-211.

Scillato-Yané, G. J., Tonni, E. P., Carlini, A. A. \& Noriega, J. I. 1998. Nuevos Hallazgos de Mamíferos del Cuaternario en el Arroyo Toropí, Corrientes, Argentina. Aspectos Bioestratigráficos, Paleoambiental y Paleozoogeograficos. X Congreso Latinoamericano de Geología y VI Congreso Nacional de Geología Económica, Actas, I, 263-268.

Scillato-Yané, G. J., Carlini, A. A., Tonni, E. P. \& Noriega, J. I. 2005. Paleobiogeography of the late Pleistocene pampatheres of South America. Journal of South American Earth Sciences, 20, 131-138.

Spillman, F.1949. Contribución a la Paleontología del Perú una mamifauna fósil de la región del río Ucayali. Museo de Historia Natural "Javier Prado", Universidad nacional Mayor de San Marcos, 1, 1-40.

Tauber, A. A. 1997. El registro de Mesotheriidae notoungulata en la provincia de Córdoba, República Argentina. Ameghiniana, 34, 125.

Tauber, A. A. 1999. Hallazgo de una vicuña en el Pleistoceno de la provincia de Córdoba, República Argentina. Ameghiniana, 36, 55-62.

Tauber, A. A. 2000. Hallazgos de Proterotheriidae (Mammalia, Litopterna) en el Pleistoceno de Córdoba, Argentina. Ameghiniana, 37, 157-162.

Tauber, A. A. \& Di Ronco, J. 2000. Nuevo hallazgo de placas ventrales de Glyptodon Owen, 1839 (Mammalia, Cingulata, Glyptodontidae) en la provincia de Córdoba, República Argentina. Boletín de la Academia Nacional de Ciencias, 64, 336-347.

Tauber, A. A. \& Di Ronco, J. 2003. Un esqueleto articulado de Mylodon sp. (Tardigrada, Mylodontidae) del Pleistoceno tardío de Córdoba, Argentina. Ameghiniana, 40, 108.

Tonni, E. P. 1992. Tapirus Brisson, 1762 (Mammalia, Perissodactyla) en el Lujanense (Pleistoceno superior-Holoceno inferior) de la provincia de Entre Ríos, República Argentina. Ameghiniana, 21, 3-8.

Tonni, E. P. \& Cione, A. L. 1999. Introduction. In: Quaternary 
of South America and Antarctic Peninsula (eds. J. Rabassa \& M. Salemme), 1, 1-3.

Tonni, E. P., \& Fidalgo, F. 1979. Consideraciones sobre los cambios climáticos durante el Pleistoceno tardío-reciente en la Provincia de Buenos Aires. Aspectos ecológicos y zoogeográficos relacionados. Ameghiniana, 15, 235 253.

Tonni, E. P. \& Scillato-Yané, G. J. 1997. Una nueva localidad con mamíferos pleistocenos en el Norte de la Argentina. Aspectos paleozoogeográficos. Congreso da Associacao Brasileira de Estudos do Quaternario e Reuniao sobre o Quaternario da America do Sul, No. 6, Anais, Curitiba, Brasil, 345-348.

Tonni, E. P., Cione, A. L. \& Bond, M. 1999a. Quaternary Vertebrate Palaeontology in Argentina. Now and then. Argentina. In: Quaternary of South America and Antarctic Peninsula (Eds. J. Rabassa \& M. Salemme). Centro Austral de Investigaciones Científicas and Universidad Nacional de la Patagonia, Ushuaia, Tierra del Fuego, 12, 5-22

Tonni, E. P., Cione, A. L., \& Figini, A. J., 1999b. Predominance of arid climates indicated by mammals in the pampas of Argentina during the Late Pleistocene and Holocene. Palaeogeography, Palaeoclimatology, Palaeoecology, 147, 257-281.

Tonni, E. P., Huarte, R., Carbonari, J. E. \& Figini, A. J. 2003. New radiocarbon chronology for the Guerrero Member of the Luján Formation (Buenos Aires, Argentina): palaeoclimatic significance. Quaternary International, 109-110, 45-48.

Tonni, E. P, Carlini, A. A, Zurita, A. E, Frechen, M, Gasparini, G., Budziak, D. \& Kruck, W. 2005. Cronología y Bioestratigrafía de la Unidades del Pleistoceno aflorantes en el Arroyo Toropí, provincia de Corrientes, Argentina. $19^{\circ}$ Congreso Brasileiro de Paleontología y $6^{\circ}$ Congreso Latino-Americano de Paleontologia, edición electrónica de resúmenes.

Ubilla, M., 1985. Mamíferos fósiles, geocronología y paleoecología de la Formación Sopas (Pleistoceno Superior.) del Uruguay. Ameghiniana, 22, 185- 196.

Ubilla, M. 1996. Paleozoología del Cuaternario de la Cuenca Norte del Uruguay: Biogeografía, Cronología y Aspectos Climáticos-Ambientales. Tesis Doctoral, Universidad de la República (Uruguay), 1-232 pp. (inédita).
Ubilla, M. \& Perea, D. 1999. Quaternary vertebrales of Uruguay: A biostratigraphic, biogeographic and climatic overview. In: Quaternary of South America and Antarctic Peninsula (Eds. J. Rabassa \& M. Salemme). Centro Austral de Investigaciones Científicas and Universidad Nacional de la Patagonia, Ushuaia, Tierra del Fuego, 6, 75-90.

Ubilla, M., Perea, D., Aguilar, C. G. \& Lorenzo, N. 2004. Late Pleistocene vertebrate from northern Uruguay: tools for biostratigraphic, climatic and environmental reconstruction. Quaternary International, 114, 129-142.

Vucetich, M. G., Noriega, J.I. \& Tonni, E.P. 2001. Hallazgo de un roedor de afinidades inciertas en el Cuaternario en la provincia de Entre Ríos (Argentina). Ameghiniana, 38, 43R.

Vucetich, M. G., Vieytes, E. C., Verzi, D. H., Noriega, J. I. \& Tonni, E. P. 2005. Unexpected primitive rodents in the Quaternary of Argentina. Journal of South American Earth Sciences, 20, 57-64.

Zurita, A. E. 2000. Primeros Registros de Gliptodontes Cuaternarios en la provincia del Chaco. FACENA, 16 , 71-84.

Zurita, A. E. 2002. Nuevo gliptodonte (Mammalia, Glyptodontoidea) del Cuaternario de la provincia de Chaco, Argentina. Ameghiniana, 39, 175-182.

Zurita, A.E. \& Carlini, A.A. 2001. Primeros registros de gliptodontes cuaternario en le provincia del Chaco. Natura Neotropicalis, 32, 69-70.

Zurita, A. E. \& Lutz, A. I. 2002. La fauna pleistocena de la Formación Toropí en la provincia de Corrientes (Argentina). Mastozoología Neotropical, 9, 47-56.

Zurita, A. E., Carlini, A. A., Scillato-Yané, G. J. \& Tonni, E. P. 2004. Mamíferos extintos del Cuaternario de la provincia del Chaco (Argentina) y su relación con aquellos del este de la región pampeana y de Chile. Revista Geológica de Chile, 31, 65-89.

Zurita, A. E., Scillato-Yané, G. J. \& Carlini, A. A. 2005. Palaeozoogeographic, biostratigraphic and systematic aspects of the genus Sclerocalyptus Ameghino 1891 (Xenarthra, Glyptodontidae) of Argentina. Journal of South American Earth Sciences, 20, 120-129.

Manuscrito recibido: 16 de Agosto, 2006 Manuscrito aceptado: 2 de abril, 2007 